\title{
Cytomegalovirus Retinitis after Multiple Ocular Surgeries in an Immunocompetent Patient
}

\author{
Koji Toriyama Takashi Suzuki Yuko Hara Yuichi Ohashi \\ Department of Ophthalmology, Ehime University School of Medicine, \\ Toon, Japan
}

\section{Key Words}

Cytomegalovirus retinitis $\cdot$ Immunocompetent patient $\cdot$ Multiple ocular surgeries

\author{
Abstract \\ Purpose: To describe the case of a 74-year-old man who developed cytomegalovirus (CMV) \\ retinitis after multiple ocular surgeries.
}

Methods: Observational case report.

Results: A 74-year-old man who had a history of multiple ocular surgeries developed unilateral retinitis with whitening of the entire peripheral retina. A presumptive diagnosis of viral retinitis was considered, and polymerase chain reaction of the aqueous fluid was positive for CMV DNA. Laboratory examination revealed that the patient was completely immunocompetent. Moreover, the patient did not have any subtenon or intravitreal injection of triamcinolone acetonide (TA). The patient responded well to intravenous ganciclovir and oral valganciclovir.

Conclusion: $\mathrm{CMV}$ retinitis can occur to immunocompetent patients without local immunosuppression with TA injection.

\section{Introduction}

Cytomegalovirus (CMV) retinitis is a viral inflammation of the retina in immunosuppressed patients, which sometimes results in severe visual loss. We report an immunocompetent patient who developed unilateral CMV retinitis after multiple ocular surgeries.

\section{Case Report}

A 74-year-old man who had a cataract surgery, 8 glaucoma surgeries for secondary glaucoma, a vitrectomy for retinal detachment, and a deep lamellar endothelial keratoplasty and 2 penetrating 
keratoplasties for bullous keratopathy for his right eye visited our clinic for a routine examination. He had used $0.05 \%$ cyclosporine eye drops once a day for over ten years after the keratoplasties. Funduscopic examination revealed areas of confluent exudate in the entire peripheral retina in his right eye (fig. 1). However, slit-lamp examination revealed no inflammatory cells in the anterior chamber or the anterior vitreous. Although the retinitis did not reach the macular area, the patient's best corrected visual acuity (OD) was hand motion because of optic nerve atrophy due to glaucoma. Fluorescein angiography demonstrated dye leakage in the corresponding peripheral retina, and severely reduced retinal blood flow due to retinal atrophy caused by multiple ocular surgeries (fig. 2 ). A presumptive diagnosis of viral retinitis was considered, with possible causative organisms including herpes simplex virus (HSV), varicella-zoster virus (VZV), or cytomegalovirus (CMV). Aqueous fluid was aspirated for use in real-time polymerase chain reaction (PCR) analysis, and prior to getting results, 1,500 mg acyclovir was administered intravenously. Real-time PCR of the aqueous fluid was positive for CMV DNA (3.89 $\times 10^{6}$ copies/ml) and negative for HSV DNA or VZV DNA. Laboratory examination revealed that the patient was negative for human immunodeficiency virus and had a normal CD4 cell count $(611 / \mu \mathrm{l})$. Immunoglobulin $\mathrm{G}$ and A were within the normal range. CMV antigenemia was negative. There were no signs of systemic CMV infection or systemic disease such as diabetes mellitus except for a history of acute myocardial infarction. Since the laboratory analyses indicated CMV retinitis, the cyclosporine eye drops were stopped and the patient was treated with $300 \mathrm{mg}$ intravenous ganciclovir twice daily for 14 days, and two intravitreal ganciclovir injections $[0.4$ $\mathrm{mg}$ ). The retinitis lesion slowly decreased in size, but real-time PCR of the aqueous fluid after intravenous ganciclovir administration revealed $9.37 \times 10^{5}$ copies/ml of CMV DNA. Thus, the patient was treated with oral valganciclovir ( $900 \mathrm{mg}$ /day) for 8 weeks. After disappearance of the retinitis, real-time PCR of aqueous specimens did not detect CMV DNA. However, a recurrence of CMV retinitis was observed two months after finishing oral ganciclovir. After two additional weeks of oral valganciclovir, the retinitis resolved (fig. 3 ). The left eye showed no CMV retinitis at any time during the follow-up period.

\section{Discussion}

CMV retinitis usually affects severely immunosuppressed individuals. However, previous studies have also reported CMV retinitis in immunocompetent patients after intravitreal or subtenon injection of triamcinolone acetonide (TA) [1-3]. Interestingly, most patients who have developed CMV retinitis after using steroids had diabetes mellitus [1-3] and it has been suggested that CMV retinitis and diabetes mellitus may be related $[3,4]$. Our patient was immunocompetent based on the results of laboratory examinations and did not have systemic disorders such as diabetes mellitus, nor any history of TA injection. CMV retinitis in immunocompetent patients typically manifests with anterior and vitreous inflammation [1-3], but no inflammatory reaction was observed in our case, similar to the typical manifestation in an immunocompromised patient. Thus, it seems likely that the immune reaction in our patient's right eye was severely suppressed. Although topical betamethasone was administered after the second keratoplasty, it was stopped about six months before the onset of the CMV retinitis. Since the patient had received cyclosporine eye drops for over ten years after the first keratoplasty, one possibility is that long-term use of cyclosporine eye drops induced local immunosuppression, leading to CMV retinitis. However, previous work shows that penetration of cyclosporine eye drops into intraocular tissues is poor [4]. Although our patient had penetrating keratoplasty for the right eye, there is few epithelial damage of the cornea in his right eye, thus it is supposed that his right eye had almost normal corneal barrier function. Moreover, since this patient exhibited recurrences of CMV retinitis even after discontinuing cyclosporine eye drops, other possibilities should be considered. Retinal vasculopathy, such as damaged retinal vascular endothelium and reduced blood flow, is thought to promote leukocyte 
entrapment in the retina and reactivation of latently infecting CMV [5, 6]. In our patient, fluorescein angiography demonstrated that retinal blood flow in his right eye was extremely decreased due to multiple ocular surgeries, and this may have some relevance to local immunosuppression.

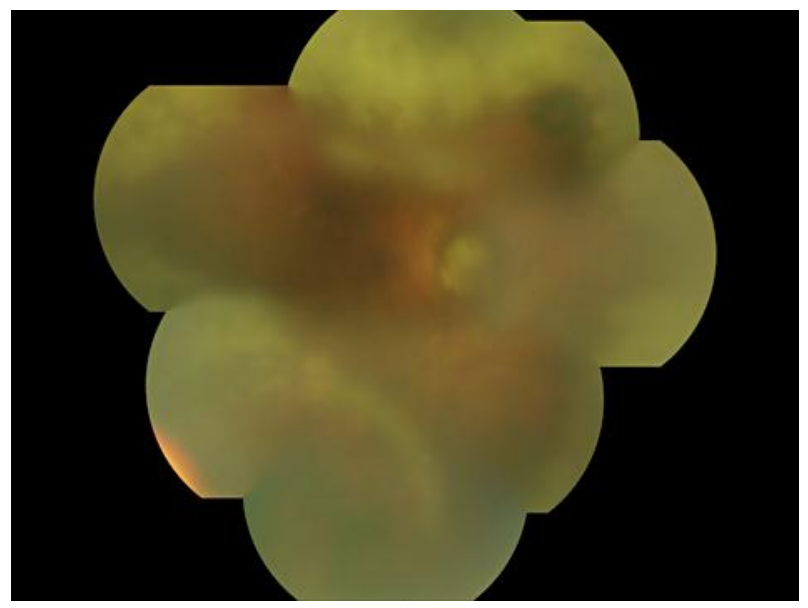

Fig. 1. Fundus photograph of the right eye. Confluent retinitis with whitening of the entire peripheral retina was observed. The optic nerve was atrophic due to glaucoma.

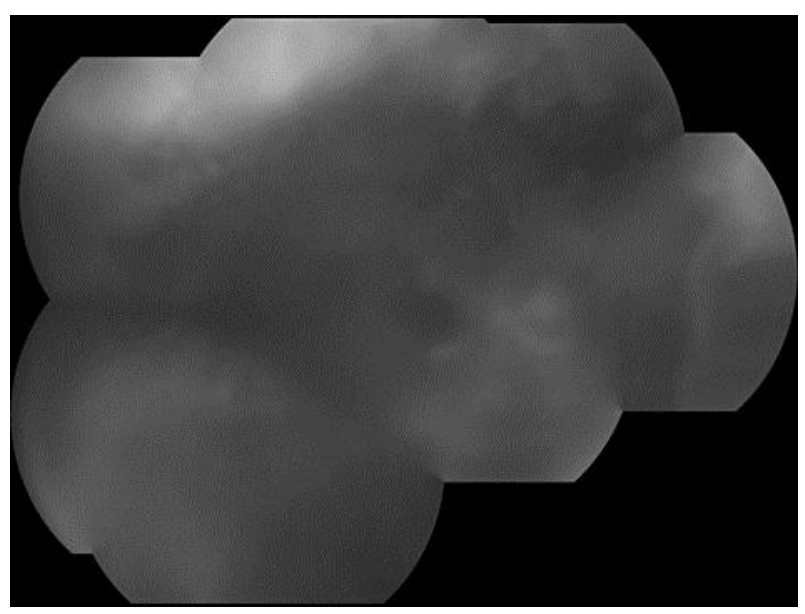

Fig. 2. Fluorescein angiography of the right eye. Dye leakage was observed corresponding with retinal white lesion, and retinal blood flow was severely reduced due to retinal atrophy caused by multiple ocular surgeries. 


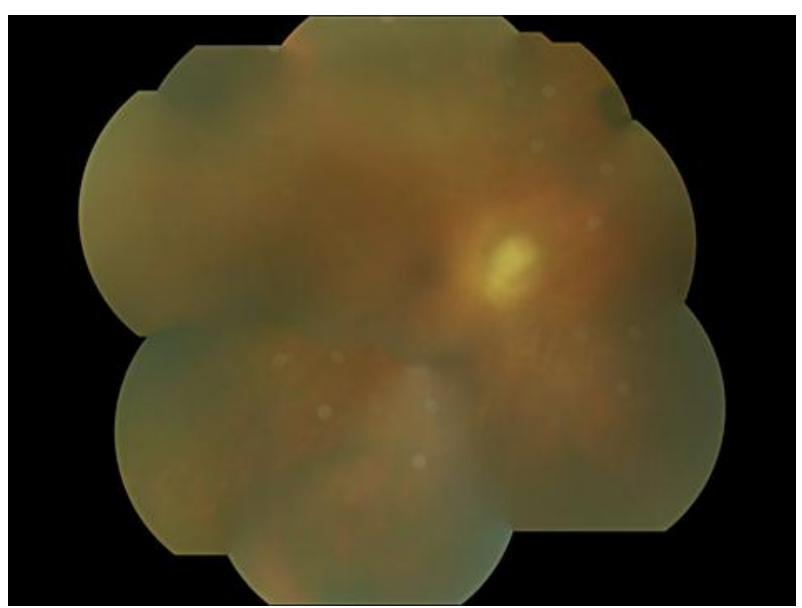

Fig. 3. Fundus photograph after treatment. The whitened retinal area had disappeared. No inflammation was observed during the follow-up period.

\section{References}

1 Delyfer MN, Rougier MB, Hubschman JP, et al: Cytomegalovirus retinitis following intravitreal injection of triamcinolone: report of two cases. Acta Ophthalmol Scand 2007;85:681-683.

$>2$ Saidel MA, Berreen J, Margolis TP: Cytomegalovirus retinitis after intravitreous triamcinolone in an immunocompetent patient. Am J Ophthalmol 2005;140:1141-1143.

-3 Toyokawa N, Kimura H, Kuroda S: Cytomegalovirus retinitis after subtenon triamcinolone acetonide and intravitreal injection of anti-vascular endothelial growth factor in an immunocompetent patient with age-related macular degeneration and diabetes mellitus. Jpn J Ophthalmol 2010;54:166-168.

4 Acheampong AA, Shackleton M, Tang-Liu DD, et al: Distribution of cyclosporin A in ocular tissues after topical administration to albino rabbits and beagle dogs. Curr Eye Res 1999;18:91-103.

5 Glasgow BJ, Weisberger AK: A quantitatibe and cartographic study of retinal microvasculopathy in acquired immunodeficiency syndrome. Am J Ophthalmol 1994;118:46-56.

6 Yoshinaga W, Mizushima Y, Abematsu N, et al: Cytomegalovirus retinitis in immunocompetent patients. J Jpn Ophthalmol Soc 2008;112:684-687. 